\title{
Nouveaux dialysats : quel acide dans le bain de dialyse ?
}

Acetate-free bicarbonate dialysate: which acid in the dialysis buffer?

Myriam Dao 1,*, Malik Touam 1, Dominique Joly 1, Lucile Mercadal 2,3

${ }^{1}$ Service de néphrologie adulte/dialyse, hôpital universitaire Necker, AP-HP, 149 rue de Sèvres, 75015 Paris.

${ }^{2}$ Service de néphrologie/dialyse, hôpital de la Pitié-Salpêtrière, AP-HP, 47-83, boulevard de l’Hôpital, 75013 Paris, France

${ }^{3}$ Inserm CESP équipe 5, épidémiologie rénale et cardiovasculaire, Villejuif

*Auteur correspondant ; e-mail : myriam.dao@aphp.fr ; tél. : 0144495458 ; fax : 0144495450

Conflit d'intérêts : aucun. 


\section{Introduction}

Dès les débuts de l'hémodialyse le problème de la précipitation irréversible du calcium et du magnésium en présence de bicarbonate s'est posé. Initialement, le bicarbonate a été remplacé dans le dialysat par de l'acétate (35-45 $\mathrm{mmol} / \mathrm{L}$ ), un précurseur du bicarbonate qui ne précipite pas en présence des cations divalents [1]. Les effets délétères de l'acétate et sa mauvaise tolérance clinique ont motivé la réintroduction du bicarbonate dans le dialysat. L'ajout de faibles doses d'acide acétique (3-7 $\mathrm{mmol} / \mathrm{L}$ ) permet l'acidification du dialysat et ainsi de prévenir la formation des précipités de bicarbonates de calcium et de magnésium. D'autres acides, l'acide chlorhydrique et plus récemment l'acide citrique, ont été proposés pour s'affranchir totalement de l'acide acétique. Deux études françaises réalisées à partir des données du registre du Réseau épidémiologie et information en néphrologie (REIN), la première publiée en 2015 et la seconde présentée en congrès par le Dr Mercadal en octobre 2018, ont mis en évidence une association entre la survie et le type de dialysat, soulignant l'importance de son rôle [2].

\section{Place de l'acide dans le dialysat}

\subsection{Composition du dialysat}

Le dialysat, fabriqué extemporanément par le générateur de dialyse, assure l'épuration des déchets et la régulation de l'équilibre électrolytique [2]. Quantitativement, les six ions les plus importants du milieu extracellulaire sont $\mathrm{Na}^{+}, \mathrm{K}^{+}, \mathrm{Ca}^{2+}, \mathrm{Mg}^{2+}, \mathrm{HCO}_{3}{ }^{-}$et $\mathrm{Cl}^{-}$. Afin de maintenir ou de restaurer l'équilibre électrolytique, ces six ions doivent être présents dans le dialysat. À pH neutre, les ions bicarbonates précipitent avec les cations divalents $\left(\mathrm{Ca}^{2+}\right.$ et $\left.\mathrm{Mg}^{2+}\right)$ et forment des précipités insolubles de carbonate de calcium et de magnésium. Les ions bicarbonates étant en large excès par rapport aux cations divalents, le risque est celui d'une importante diminution de la concentration de ces cations divalents. L'acidification du dialysat par adjonction de $\mathrm{CO}_{2}$ dissous prévient la formation de ces précipités insolubles. Plusieurs approches ont été développées depuis les débuts de l'hémodialyse afin de répondre à cette problématique.

\subsection{De la dialyse à l'acétate à la dialyse au bicarbonate}

La dissolution directe de $\mathrm{CO}_{2}$ gazeux dans le dialysat était malaisée. Une première réponse a été proposée par Charles Mion et Belding Scribner en 1964 avec le remplacement du bicarbonate par de l'acétate [1]. L'acétate est facilement utilisable en solution, ne précipite pas avec les cations divalents et est secondairement métabolisé en bicarbonate. Cependant, en l'absence de bicarbonate dans le dialysat, de fortes concentrations d'acétate ( 35 à $45 \mathrm{mmol} / \mathrm{L}$ ) sont nécessaires afin d'une part de permettre la régénération des bicarbonates consommés durant l'intervalle inter-dialytique, d'autre part de compenser la perte per-dialytique de bicarbonates liée à l'absence de celui-ci dans le dialysat [1]. Le taux d'acétate transféré durant les séances d'hémodialyse est alors proche du taux maximal de 
conversion de l'acétate en bicarbonates, évalué à 3-3,5 $\mathrm{mmol} / \mathrm{kg} / \mathrm{h}$. Après une séance d'hémodialyse à l'acétate, l'acétatémie peut s'élever jusqu'à 15 mmol/L [3-6], ce qui est très supérieur à sa concentration physiologique $(<0.15 \mathrm{mmol} / \mathrm{L})$ [7-9]. Les conséquences cliniques de cette hyperacétatémie sont nombreuses : instabilité hémodynamique en rapport avec une vasodilatation périphérique et une altération de la contractilité myocardique, bio-incompatibilité et inflammation, acidose [6,10-15]. Une seconde avancée a été la réintroduction du bicarbonate comme composé majoritaire du dialysat. L'ajout d'acide acétique à faible concentration (3-7 $\mathrm{mmol} / \mathrm{L})$ permet d'acidifier le dialysat et prévient ainsi la précipitation du bicarbonate avec les cations divalents. La généralisation de l'hémodialyse au bicarbonate dans les années 1980 s'est accompagnée d'une nette amélioration de la tolérance des séances, notamment hémodynamique $[3,10,11,16]$. Il semble persister malgré tout une augmentation significative de l'acétatémie après les séances d'hémodialyse, bien que les études ne soient pas toutes concordantes : une étude récente a trouvé une concentration normale d'acétate [17], à l'inverse de travaux antérieurs qui montraient une élévation de la concentration d'acétate de 10 à 70 fois la limite supérieure de la normale [18,19].

\subsection{Métabolisme de l'acide acétique}

En présence de bicarbonate, l'acide acétique contenu dans le dialysat se transforme en acétate et libère du $\mathrm{CO}_{2}$ dissous selon la réaction suivante : $\mathrm{CH}_{3} \mathrm{COOH}+\mathrm{HCO}_{3}{ }^{-} \rightarrow \mathrm{CH}_{3} \mathrm{COO}^{-}+\mathrm{CO}_{2}+\mathrm{H}_{2} \mathrm{O}$. Arrivé dans l'organisme, l'acétate est transformé en bicarbonate par le foie et les muscles. Une partie de l'acétate se conjugue au coenzyme A (Figure 1). Cette conjugaison nécessite la transformation d'ATP en AMP cyclique (AMPc) par l'adénylate cyclase. L'AMPc est le second messager de l'action de nombreuses hormones, dont des protéines kinases AMPc-dépendantes (PKA) dont il démasque l'activité phosphorylante (20). L'AMPc active la NO synthase qui catalyse la transformation de Larginine en L-citrulline + NO, notamment dans les cellules endothéliales [21]. In vitro, l'incubation de cellules endothéliales avec différentes concentrations d'acétate augmente l'activité de la $\mathrm{N}$ synthase ainsi que sa transcription, renforçant cette hypothèse [22]. La sécrétion d'interleukine 1 (IL-1), impliquée dans l'inflammation, l'activation des cellules lymphocytaires et la stimulation de la NO synthase inductible, est également augmentée en cas d'exposition à l'acétate, tant in vitro qu'in vivo $[13,15]$. Les effets cliniques observés lorsque le seuil de conversion de l'acétate en bicarbonate est dépassé sont donc au moins en partie médiés par la sécrétion de NO et de médiateurs de l'inflammation dont l'interleukine 1 (IL-1).

\section{Acide chlorhydrique}

Dès la fin du $20^{\mathrm{e}}$ siècle, l'acide chlorhydrique $(\mathrm{HCl})$ a été proposé en remplacement de l'acide acétique pour acidifier le dialysat [19]. L'ion $\mathrm{Cl}^{-}$assure l'électroneutralité dans l'organisme ; il présente l'avantage de n'être soumis à aucune boucle de régulation. Sa concentration est fixée dès lors 
que celles des autres ions sont fixées [23]. L'utilisation d'HCl en remplacement de l'acide acétique permet donc théoriquement de s'affranchir des complications métaboliques engendrées par l'acétate. La principale difficulté est d'ordre technique. La forte acidité de l'HCl engendre des contraintes de fabrication (cuves et tuyauteries en acier inoxydable), de conditionnements (bidons et poches résistants à l'acidité) et d'utilisation (circuits hydrauliques des générateurs d'hémodialyse), à l'origine d'un surcoût [23]. Une solution pour rendre le concentré moins agressif, proposée par certains fabricants, consiste à réduire la concentration d' $\mathrm{HCl}$ à $2 \mathrm{mmol} / \mathrm{L}$. La concentration de $\mathrm{CO}_{2}$ dissous s'en trouve alors également réduite, ce qui augmente le risque de précipitation de bicarbonate de calcium et nécessite une décalcification plus fréquente des générateurs. Le bénéfice clinique de la dialyse à l'HCl a été confirmé dans une étude française basée sur les données du registre du REIN publiée en 2015 par Mercadal et al. [2]. Les auteurs ont classé 15160 patients incidents ayant démarré l'hémodialyse de suppléance entre 2008 et 2010 en trois catégories selon le type de dialysat utilisé dans leur centre : 11481 patients n'avaient jamais été exposé à un dialysat à l'HCl, 3021 étaient dialysés dans une unité dite mixte utilisant des dialysats au bicarbonate acidifié à l'acide acétique et des dialysats à l' $\mathrm{HCl}$ et 658 avaient été dialysés au moins une fois dans une unité utilisant exclusivement des dialysats à l'HCl. Chez les patients âgés de plus de 70 ans, la dialyse sans acétate était associée à une amélioration de la survie, indépendamment des comorbidités et du recours à l'hémodiafiltration. Le surcoût engendré par les contraintes techniques et l'arrivée sur le marché des dialysats au bicarbonate acidifiés à l'acide citrique ont probablement freiné la généralisation des dialysats à l’ $\mathrm{HCl}$.

\section{Acide citrique}

\subsection{Métabolisme du citrate}

Le citrate, dont la concentration plasmatique normale est d'environ $0,1 \mathrm{mmol} / \mathrm{L}$, est un intermédiaire du cycle de Krebs. Le rein est un acteur majeur de son métabolisme [24-27]. Le citrate est librement filtré au niveau glomérulaire. Une part importante (65-90\%) est réabsorbée activement au niveau du tubule proximal, le reste (10-35\%) étant excrété dans l'urine. Néanmoins, Bauer et al. ont montré que la clairance du citrate et son métabolisme à long-terme n'étaient pas altérés en cas d'insuffisance rénale chronique [28]. En effet, le citrate peut être oxydé par les cellules hépatiques ou musculaires selon la réaction suivante : $\mathrm{Ca}_{3}\left(\mathrm{C}_{6} \mathrm{H}_{5} \mathrm{O}_{7}\right)_{2}+9 \mathrm{O}_{2} \rightarrow 6 \mathrm{CO}_{2}+6 \mathrm{HCO}_{3}{ }^{-}+3 \mathrm{Ca}^{2+}+2 \mathrm{H}_{2} \mathrm{O}$, libérant ainsi trois molécules de bicarbonate par molécule de citrate et rendant possible son utilisation en cas d' insuffisance rénale chronique [23]. Des études menées en réanimation ont montré une bonne tolérance en aigu de l'anticoagulation régionale au citrate chez des patients présentant non seulement une insuffisance rénale mais également une insuffisance hépatique [29-31]. Une surveillance rapprochée de ces patients reste néanmoins indispensable : Meier-Kriesche et al. ont rapporté le cas d'une patiente de 71 ans, hospitalisée pour une hépatite fulminante et hémodialysée avec une anticoagulation régionale au citrate, qui a présenté une hypocalcémie ionisée profonde associée à une élévation de la 
calcémie totale, suggérant une accumulation du citrate [32]. Il n'existe pas d'étude similaire chez des patients hémodialysés chroniques ayant une insuffisance hépatique.

\subsection{Rationnel de l'utilisation de l'acide citrique en hémodialyse}

Le principe de l'hémodialyse à l'acide citrique $\mathrm{H}_{3} \mathrm{C}_{6} \mathrm{H}_{5} \mathrm{O}_{7}$ consiste à remplacer en partie, voire en totalité, l'acide acétique dans le dialysat par de l'acide citrique. Les propriétés anticoagulantes du citrate et une meilleure biocompatibilité de l'acide citrique par rapport à l'acide acétique ont guidé ce choix. L'ion citrate trivalent chélate le calcium, réduisant ainsi la coagulation et l'activation du complément $[33,34]$. En outre, des travaux expérimentaux ont mis en évidence une réduction du stress oxydatif par le citrate. Cet effet serait médié par la chélation d'ions multivalents (fer, cuivre), la restauration du glutathion mitochondrial, l'inhibition de la formation de radicaux libres [35,36]. Les intérêts potentiels de l'acide citrique sont donc multiples : la dialyse sans héparine, l'amélioration de la perméabilité de la membrane grâce à l'action anticoagulante de l'acide citrique, la diminution des phénomènes inflammatoires, une meilleure stabilité hémodynamique en séance, le contrôle de l'acidose [37-40]. La mise sur le marché des dialysats ne répondant pas au même processus que celle des médicaments, les études présentent des schémas et des méthodologies très divers rendant parfois malaisée leur comparaison (Tableau 1).

\subsection{Effets de l'acide citrique sur l'épuration}

La première étude comparant un dialysat au bicarbonate acidifié à l'acide citrique et un dialysat au bicarbonate acidifié à l'acide acétique en hémodialyse chronique a été rapportée par Ahmad et al. [38] en 2000 aux Etats-Unis. Vingt-deux patients de 55,5 \pm 13,1 ans, dialysés depuis 7,3 \pm 4,7 ans, ont été hémodialysés pendant 12 semaines avec un dialysat au bicarbonate acidifié à l'acide citrique. Les auteurs ont comparé les données de la première et la dernière séance de dialyse. Le Kt/V calculé et le taux de réduction de l'urée ont significativement augmenté durant l'étude, passant respectivement de $1,23 \pm 0,19$ à $1,34 \pm 0,20(\mathrm{p}=0,01)$ et de $68 \% \pm 5,9 \%$ à $73 \% \pm 5,3 \%(\mathrm{p}<0,03)$. Les débits du sang et du dialysat, la durée de dialyse et les membranes n'ont pas été modifiés pendant l'étude. Les auteurs émettent l'hypothèse que l'action anticoagulante locale du citrate préserve la perméabilité de la membrane, améliorant ainsi la qualité de l'épuration. Des résultats similaires ont été rapportés par Kossmann et al. dans une étude comparant les résultats de 6 mois d'hémodialyse avec un dialysat au bicarbonate acidifié à l'acide citrique par rapport aux 6 mois précédents avec un dialysat au bicarbonate acidifié à l'acide acétique ( $\mathrm{n}=142$, augmentation significative $\mathrm{du} \mathrm{Kt} / \mathrm{V}$, diminution significative de la $\beta 2$-microglobulinémie) [37]. Ces dernières années, des études prospectives randomisées en cross-over ont été réalisées et n'ont pas permis de mettre en évidence de différence dans les paramètres d'épuration entre l'acide citrique ou l'acide acétique [40-42]. L'amélioration des membranes de dialyse et notamment l'utilisation de membrane à usage unique dans les études les plus récentes pourrait expliquer ces résultats [42]. Très récemment, deux études se sont intéressées à la 
clairance de l'indoxyl sulfate, une toxine urémique de petit poids moléculaire $(213 \mathrm{~g} / \mathrm{mol})$ dérégulant l'expression de près de 2000 gènes in vitro, au cours de dialyse utilisant l'acide citrique ou l'acide acétique [43-45]. Dans l'étude de Hyšpler et al., la réduction per-dialytique d'indoxyl sulfate était supérieure lors de dialyse avec un dialysat au bicarbonate acidifié à l'acide acétique [43]. Dans l'étude de Jung et al., l'épuration de l'indoxyl sulfate était similaire entre les deux types de dialysats [44]. En revanche, les dommages cellulaires et l'inflammation étaient moindres lors de la dialyse utilisant l'acide citrique. Les auteurs ont émis l'hypothèse que l'inhibition du métabolisme lié au calcium expliquait la diminution du syndrome inflammatoire. Le calcium étant impliqué dans de nombreuses voies métaboliques, la question de savoir si l'inhibition des métabolismes dépendants du calcium n'est que bénéfique reste ouverte.

\subsection{Effets de l'acide citrique sur l'anticoagulation}

Les propriétés anticoagulantes du citrate reposent sur sa capacité à chélater le calcium ionisé, cofacteur essentiel de plusieurs étapes de la cascade de coagulation. L'utilisation d'héparine en hémodialyse n'est pas dénuée de risques, qu'il s'agisse d'événements hémorragiques ou de complications immunologiques (thrombopénie auto-immune, réactions anaphylactiques en rapport avec des contaminants tels que le sulfate de chondroïtine sursulfaté) $[46,47]$. Dans ce contexte, plusieurs équipes ont étudié l'effet d'une réduction voire d'une suppression de l'héparine lors de séances d'hémodialyse avec un dialysat au bicarbonate acidifié à l'acide citrique [48-50]. Dans une étude prospective conduite chez 277 patients, Sands et al. ont rapporté une qualité d'épuration similaire avec un dialysat au bicarbonate acidifié à l'acide acétique et 100\% de la dose d'héparine habituelle du patient, ou un dialysat au bicarbonate acidifié à l'acide citrique et soit $100 \%$, ou $80 \%$, ou $66,7 \%$ de la dose d'héparine habituelle du patient (Kt/V respectivement 1,548 $\pm 0,29,1,548 \pm 0,28,1,558 \pm 0,27$ et $1,548 \pm 0,26)$ [48]. Des résultats similaires ont été obtenus dans une étude prospective, randomisée, en cross-over, comparant 12 semaines d'hémodialyse avec un dialysat au bicarbonate acidifié à l'acide acétique ou avec un dialysat au bicarbonate acidifié à l'acide citrique et 30\% d'héparine en moins [49]. Plus récemment, dans une étude prospective, randomisée, en cross-over et en double-aveugle, Leung et al. ont comparé 2 semaines d'hémodialyse avec un dialysat au bicarbonate acidifié à l'acide citrique ou avec un dialysat au bicarbonate acidifié à l'acide acétique avec diminution en aveugle des doses d'héparine [50]. Dans les deux groupes, la dose d'héparine a pu être significativement diminuée (19\% avec l'acide acétique, 30\% avec l'acide citrique) mais la moyenne de réduction n'était pas significativement différente entre les groupes, peut-être en raison d'un manque de puissance lié à l'effectif $(n=20)$, calculé à partir des résultats des études antérieures $[48,49]$. L'absence d'évaluation de la diminution de l'héparine avec l'acidification du dialysat à l'acide acétique dans ces deux études antérieures a pu conduire à surestimer l'effet de l'acide citrique dans la réduction de l'héparine. 


\subsection{Effets de l'acide citrique sur la balance calcique}

La chélation du calcium par le citrate est en théorie transitoire : une fois arrivé dans l'organisme, le métabolisme rapide du citrate permet de libérer le calcium. Cependant, toutes les études ont mis en évidence un impact de l'utilisation de dialysat au bicarbonate acidifié à l'acide citrique sur la calcémie ionisée avec de façon constante une diminution significative de la calcémie ionisée au cours de la séance d'hémodialyse [38,40-42,48-51]. Une augmentation de la concentration de calcium dans le dialysat de 0,15 à $0,25 \mathrm{mmol} / \mathrm{L}$ est nécessaire avec le citrate par rapport à l'acétate pour maintenir des niveaux équivalents de calcémie ionisée [40,51]. Le comportement perdialytique de la parathormone (PTH) est également modifié par l'utilisation de dialysat au bicarbonate acidifié à l'acide citrique. Avec un dialysat au bicarbonate acidifié à l'acide citrique contenant $1,5 \mathrm{mmol} / \mathrm{L}$ de calcium, la PTH ne varie pas au cours de la séance de dialyse, alors qu'elle diminue significativement en cas d'utilisation d'un dialysat au bicarbonate acidifié à l'acide acétique contenant la même concentration de calcium [41,42,51]. Des données récentes chez 126 patients dans une étude en cross-over ont montré qu'alors que la PTH diminuait de $64 \%$ au cours d'une dialyse à l'acétate (de 22,2 [15,3;36,3] à $8,0[5,9 ; 12,5] \mathrm{pmol} / \mathrm{L}, \mathrm{p}<0,0001)$, elle avait tendance à augmenter de $4 \%$ lors de l'utilisation de citrate (de 27,0 [20,6; 36,9] à 28,0 [21,5; 41,2] pmol/L, p=0,06) [51]. Une augmentation de plus de $25 \%$ était observée dans $28 \%$ des dialyses au citrate. Comme attendu, les variations de la PTH étaient inversement corrélées avec les variations de la calcémie ionisée. L’impact à long terme de ces variations de la PTH sur le métabolisme minéral et osseux reste à préciser.

\subsection{Effets hémodynamiques de l'acide citrique}

L'utilisation de citrate est associée de façon assez constante à une réduction de la pression artérielle, médiée en partie par une diminution des résistances vasculaires périphériques [40,41,49]. Gabutti et al. ont montré que par rapport à la dialyse à l'acétate, pour une même concentration de calcium dans le dialysat $(1,5 \mathrm{mmol} / \mathrm{L})$, la dialyse au citrate est associée à une diminution plus importante de la pression artérielle, tant systolique que diastolique (respectivement $-4,3$ et $-1,6 \mathrm{mmHg}, \mathrm{p}<0,01$ ) et des résistances périphériques ( $\mathrm{p}<0,001)$, sans modification du volume d'éjection systolique (augmentation non significative de $5 \mathrm{~mL}$ ) [40]. Après augmentation de $0,25 \mathrm{mmol} / \mathrm{L}$ de la concentration de calcium dans le dialysat au bicarbonate acidifié à l'acide citrique : la pression artérielle retrouvait un niveau similaire à celle observée au cours de la dialyse à l'acétate, le volume d'éjection systolique était préservé mais la diminution des résistances périphériques s'accentuait, traduisant une moindre vasoconstriction. Les auteurs soulignent cependant qu'au niveau individuel, les variations tensionnelles les plus importantes étaient observées au cours de dialyse à l'acétate. 


\subsection{Tolérance clinique}

Plusieurs auteurs ont rapporté une augmentation de la fréquence des crampes avec le citrate, particulièrement au cours des deux premières semaines de traitement $[42,48,49]$. Une seule étude japonaise a montré une meilleure tolérance clinique du citrate par rapport à l'acétate mais la concentration d'acide acétique dans le dialysat au bicarbonate acidifié à l'acide acétique était plus élevée $(11 \mathrm{mEq} / \mathrm{L})$ et le poids des patients plus faible $(59 \mathrm{~kg})$ que dans les études européennes ou nord-américaines [52]. La diminution significative de la calcémie ionisée et de la magnésémie pourrait pour partie participer à l'augmentation de fréquence des crampes. Un défaut de métabolisation du citrate est également suspecté. Dans une étude de Durand et al., chez trois patients atteints de crampes particulièrement sévères lors de dialyse avec un dialysat au bicarbonate acidifié à l'acide citrique, la calcémie ionisée et la magnésémie diminuaient modérément et restaient dans les normes, au contraire d'une franche hypercitratémie qui s'aggravait en séance $(79 \mathrm{mg} / \mathrm{L}$ après $4 \mathrm{~h}$ de dialyse contre $37 \mathrm{mg} / \mathrm{L}$ avant la séance) [53]. Ces mêmes auteurs ont récemment comparé dix patients sous dialysat au bicarbonate acidifié à l'acide citrique, dont cinq ayant des crampes et cinq n'en ayant pas : l'apparition des crampes sous citrate semblait liée à une dysfonction mitochondriale et à une dysbiose du microbiote [54].

\subsection{Citrate et équilibre acidobasique}

Les effets délétères de l'acidose, notamment sur le métabolisme protéique, musculaire et osseux, sont bien connus. Les modalités de correction de l'acidose chez l'hémodialysé chronique restent néanmoins débattues, faute d'études suffisantes [55,56]. La Kidney Disease Outcomes Quality Initiative (KDOQI) recommande un objectif de réserve alcaline prédialytique de $22 \mathrm{mmol} / \mathrm{L}$. Selon les études, la réserve alcaline prédialytique est supérieure, identique ou au contraire inférieure en cas d'utilisation de citrate par rapport à l'acétate $[37,38,40,42,48,50]$. Il semble que le citrate entraine moins d'alcalose postdialytique que l'acétate puisque la réserve alcaline à la fin de la séance est tantôt comparable tantôt inférieure $[38,40,42,49,50]$. Compte tenu de ces résultats contradictoires et en l'absence d'études dédiées, il est difficile de conclure actuellement quant à un éventuel effet bénéfique ou au contraire défavorable du citrate par rapport à l'acétate sur l'équilibre acidobasique.

\subsection{Nutrition}

L'altération du statut nutritionnel, fréquente chez les patients hémodialysés, est associée à un pronostic défavorable. Une seule étude japonaise s'est intéressée aux conséquences du citrate sur le statut nutritionnel des patients [57]. L'état nutritionnel des patients dénutris, évalués par l'albuminémie, s'amélioraient lors du passage de l' acétate vers le citrate et s'altéraient de nouveau lors du passage du citrate vers l' acétate dans le dialysat. Les résultats sont là encore difficiles à généraliser en l'absence d'évaluation clinique de l'état nutritionnel (pas de données concernant les variations du poids sec ou 
du pourcentage de masse maigre des patients) et du fait de la concentration élevée d'acétate (10 $\mathrm{mEq} / \mathrm{L})$ dans le dialysat.

\subsection{Biocompatibilité}

L'inflammation et le stress oxydatif participent à l'augmentation de la mortalité cardiovasculaire chez les patients hémodialysés. Les résultats des études quant à l'effet de l'acide citrique sont discordants. Dans une étude japonaise, les auteurs mettent en évidence une diminution des produits de la glycoxydation et de la peroxydation des lipides, marqueurs du stress oxydatif, au cours de dialyse au citrate par rapport à l' acétate [39]. Dans l'étude de Grundström au contraire, seule l'augmentation de la pentraxin-3 était moins importante pendant la séance de dialyse. Les valeurs prédialytiques de pentraxin-3, protéine C réactive (CRP), IL-6, tumour necrosis factor (TNF)- $\alpha$ et de l' IL-1 $\beta$ étaient indépendantes du type de dialysat, de même que l'élévation perdialytique de CRP, IL-6, TNF- $\alpha$ and IL-1 [41]. Il est possible que le faible impact du type de dialysat sur les paramètres de l'inflammation et du stress oxydatif dans cette étude soit lié à la concentration en acétate ( $3 \mathrm{mmol} / \mathrm{L})$ beaucoup plus faible que celle utilisée dans l'étude japonaise $(11 \mathrm{mmol} / \mathrm{L})$ [39,41]. Les données disponibles à ce jour ne permettent donc pas de conclure sur une éventuelle supériorité du citrate dans le dialysat par rapport à un dialysat au bicarbonate à faible concentration d'acide acétique.

\subsection{Acide citrique en hémodiafiltration}

L' hémodiafiltration augmente l'exposition du patient au dialysat, notamment en postdilution, en raison des hauts volumes de substitution [58]. L'utilisation de citrate en hémodiafiltration n'augmente pas significativement le risque de saignement par rapport à l' acétate et n'induit pas plus d'hypocalcémie qu'en hémodialyse conventionnelle avec le citrate [41,51,59-61]. Le citrate est supérieur à l' acétate dans la plupart des études concernant la qualité de l'épuration [60,61]. Aniort et al. ont montré qu'il était le plus souvent possible d'arrêter l'anticoagulation par héparine en hémodiafiltration postdilution avec le citrate, sans que le Kt/V, la réserve alcaline prédialytique et la $\beta 2$-microglobuline ne soient altérés par rapport à l' acétate avec héparine [59]. Plus récemment, des résultats opposés ont été rapportés par Richova et al. en hémodiafiltration prédilution : les séances sans héparine étaient techniquement faisables mais étaient associées à une diminution significative du Kt/V et de la biocompatibilité par rapport à l' hémodiafiltration prédilution à l' acétate [62].

\section{Conclusion et perspectives}

L'hémodialyse au bicarbonate, rendue possible par l'acidification du dialysat qui prévient la précipitation des cations divalents avec le bicarbonate, s'est accompagnée d'une amélioration de la tolérance. La présence de l'acide acétique à une concentration supraphysiologique n'est néanmoins pas dénuée d'effets indésirables. L'acide chlorhydrique a été proposé en remplacement mais les contraintes techniques ont freiné son développement. Plus récemment, l'acide citrique a été proposé 
comme candidat au remplacement de l'acide acétique. Les résultats des études sont néanmoins contradictoires et de nombreuses questions, notamment quant à son impact sur le métabolisme phosphocalcique et acidobasique, restent ouvertes. À la lumière des données actuelles, il ne semble pas raisonnable de proposer le remplacement systématique de l'acide acétique par l'acide citrique. Toutes les techniques présentées ci-dessus nécessitent l'acidification du dialysat. Du fait de l'augmentation de la concentration de $\mathrm{CO}_{2}$ dissous, qui sera rapidement éliminé par voie respiratoire, le sang est plus acide à la sortie du dialyseur qu'à l'entrée, ce qui active la synthèse de bradykinines et de NO. Dans ce contexte, le développement des techniques sans acétate ni acide garde toute sa place.

\section{Conflits d'intérêt : aucun}

\section{Bibliographie}

1. Mion CM, Hegstrom RM, Boen ST, Scribner BH. Substitution of sodium acetate for sodium bicarbonate in the bath fluid for hemodialysis. Am Soc Artif Intern Organs. 1964;10:110-5.

2. Mercadal L, Franck J-E, Metzger M, Yuan W, Kolko A, Monnet E, et al. Improved survival associated with acetate-free haemodialysis in elderly: a registry-based study. Nephrol Dial Transplant. 2015 Sep;30(9):1560-8.

3. Novello A, Kelsch RC, Easterling RE. Acetate intolerance during hemodialysis. Clin Nephrol. 1976 Jan;5(1):29-32.

4. Pagel MD, Ahmad S, Vizzo JE, Scribner BH. Acetate and bicarbonate fluctuations and acetate intolerance during dialysis. Kidney Int. 1982 Mar;21(3):513-8.

5. Weiner MW. Acetate metabolism during hemodialysis. Artif Organs. 1982 Nov;6(4):370-7.

6. Vinay P, Prud'Homme M, Vinet B, Cournoyer G, Degoulet P, Leville M, et al. Acetate metabolism and bicarbonate generation during hemodialysis: 10 years of observation. Kidney Int. 1987 May;31(5):1194-204.

7. Richards RH, Dowling JA, Vreman HJ, Feldman C, Weiner MW. Acetate levels in human plasma. Proc Clin Dial Transplant. 1976 Nov 19;6:73-9.

8. Tollinger CD, Vreman HJ, Weiner MW. Measurement of acetate in human blood by gas chromatography: effects of sample preparation, feeding, and various diseases. Clin Chem. 1979 Oct;25(10):1787-90.

9. Kveim M, Bredesen JE. A gas chromatographic method for determination of acetate levels in body fluids. Clin Chim Acta Int J Clin Chem. 1979 Feb 15;92(1):27-32. 
10. Vincent JL, Vanherweghem JL, Degaute JP, Berré J, Dufaye P, Kahn RJ. Acetate-induced myocardial depression during hemodialysis for acute renal failure. Kidney Int. 1982 Dec;22(6):653-7.

11. Herrero JA, Trobo JI, Torrente J, Torralbo A, Tornero F, Cruceyra A, et al. Hemodialysis with acetate, DL-lactate and bicarbonate: a hemodynamic and gasometric study. Kidney Int. 1994 Oct;46(4):1167-77.

12. Liang CS, Lowenstein JM. Metabolic control of the circulation. Effects of acetate and pyruvate. J Clin Invest. 1978 Nov;62(5):1029-38.

13. Noris M, Todeschini M, Casiraghi F, Roccatello D, Martina G, Minetti L, et al. Effect of acetate, bicarbonate dialysis, and acetate-free biofiltration on nitric oxide synthesis: implications for dialysis hypotension. Am J Kidney Dis. 1998 Jul;32(1):115-24.

14. Todeschini M, Macconi D, Fernández NG, Ghilardi M, Anabaya A, Binda E, et al. Effect of acetate-free biofiltration and bicarbonate hemodialysis on neutrophil activation. Am J Kidney Dis. 2002 Oct;40(4):783-93.

15. Bingel M, Lonnemann G, Koch KM, Dinarello CA, Shaldon S. Enhancement of in-vitro human interleukin-1 production by sodium acetate. Lancet. 1987 Jan 3;1(8523):14-6.

16. Graefe U, Milutinovich J, Follette WC, Vizzo JE, Babb AL, Scribner BH. Less dialysisinduced morbidity and vascular instability with bicarbonate in dialysate. Ann Intern Med. 1978 Mar;88(3):332-6.

17. Smith WB, Gibson S, Newman GE, Hendon KS, Askelson M, Zhao J, et al. The dynamics of the metabolism of acetate and bicarbonate associated with use of hemodialysates in the ABChD trial: a phase IV, prospective, single center, single blind, randomized, cross-over, two week investigation. BMC Nephrol. 2017 Aug 29;18(1):273.

18. Veech RL. The untoward effects of the anions of dialysis fluids. Kidney Int. 1988 Nov;34(5):587-97.

19. Fournier G, Potier J, Thébaud HE, Majdalani G, Ton-That H, Man NK. Substitution of acetic acid for hydrochloric acid in the bicarbonate buffered dialysate. Artif Organs. 1998 Jul;22(7):608-13.

20. Krebs EG, Beavo JA. Phosphorylation-dephosphorylation of enzymes. Annu Rev Biochem. 1979;48:923-59.

21. García-Morales V, Luaces-Regueira M, Campos-Toimil M. The cAMP effectors PKA and Epac activate endothelial NO synthase through PI3K/Akt pathway in human endothelial cells.

Biochem Pharmacol. 2017 01;145:94-101. 
22. Amore A, Cirina P, Mitola S, Peruzzi L, Bonaudo R, Gianoglio B, et al. Acetate intolerance is mediated by enhanced synthesis of nitric oxide by endothelial cells. J Am Soc Nephrol. 1997 Sep;8(9):1431-6.

23. Petitclerc T, Diab R, Le Roy F, Mercadal L, Hmida J. Hémodialyse sans acétate : qu'est-ce vraiment ? Nephrol Ther. 2011 Apr;7(2):92-8.

24. Chappell JB. The oxidation of citrate, isocitrate and cis-aconitate by isolated mitochondria. Biochem J. 1964 Feb;90(2):225-37.

25. Baruch SB, Burich RL, Eun CK, King VF. Renal metabolism of citrate. Med Clin North Am. 1975 May;59(3):569-82.

26. Minisola S, Rossi W, Pacitti MT, Scarnecchia L, Bigi F, Carnevale V, et al. Studies on citrate metabolism in normal subjects and kidney stone patients. Miner Electrolyte Metab. 1989;15(5):303-8.

27. Grases F, Conte A, March JG, García-Ferragut L. Evolution of lithogenic urinary parameters with a low dose potassium citrate treatment. Int Urol Nephrol. 1998;30(1):1-8.

28. Bauer E, Derfler K, Joukhadar C, Druml W. Citrate kinetics in patients receiving long-term hemodialysis therapy. Am J Kidney Dis. 2005 Nov;46(5):903-7.

29. Schultheiß C, Saugel B, Phillip V, Thies P, Noe S, Mayr U, et al. Continuous venovenous hemodialysis with regional citrate anticoagulation in patients with liver failure: a prospective observational study. Crit Care. 2012 Aug 22;16(4):R162.

30. Klingele M, Stadler T, Fliser D, Speer T, Groesdonk HV, Raddatz A. Long-term continuous renal replacement therapy and anticoagulation with citrate in critically ill patients with severe liver dysfunction. Crit Care. 2017 Nov 29;21(1):294.

31. Rodriguez K, Srivaths PR, Tal L, Watson MN, Riley AA, Himes RW, et al. Regional citrate anticoagulation for continuous renal replacement therapy in pediatric patients with liver failure. PloS One. 2017;12(8):e0182134.

32. Meier-Kriesche HU, Finkel KW, Gitomer JJ, DuBose TD. Unexpected severe hypocalcemia during continuous venovenous hemodialysis with regional citrate anticoagulation. Am J Kidney Dis. 1999 Apr;33(4):e8.

33. Borensztajn K, Peppelenbosch MP, Spek CA. Factor Xa: at the crossroads between coagulation and signaling in physiology and disease. Trends Mol Med. 2008 Oct;14(10):429-40. 
34. Nilsson B, Ekdahl KN, Mollnes TE, Lambris JD. The role of complement in biomaterialinduced inflammation. Mol Immunol. 2007 Jan;44(1-3):82-94.

35. Bryland A, Wieslander A, Carlsson O, Hellmark T, Godaly G. Citrate treatment reduces endothelial death and inflammation under hyperglycaemic conditions. Diab Vasc Dis Res. 2012 Jan;9(1):42-51.

36. Paim BA, Velho JA, Castilho RF, Oliveira HCF, Vercesi AE. Oxidative stress in hypercholesterolemic LDL (low-density lipoprotein) receptor knockout mice is associated with low content of mitochondrial NADP-linked substrates and is partially reversed by citrate replacement. Free Radic Biol Med. 2008 Feb 1;44(3):444-51.

37. Kossmann RJ, Gonzales A, Callan R, Ahmad S. Increased efficiency of hemodialysis with citrate dialysate: a prospective controlled study. Clin J Am Soc Nephrol. 2009 Sep;4(9):1459-64.

38. Ahmad S, Callan R, Cole JJ, Blagg CR. Dialysate made from dry chemicals using citric acid increases dialysis dose. Am J Kidney Dis. 2000 Mar;35(3):493-9.

39. Masuda A, Hagiwara S, Tanimoto M, Kodama F, Okumura K, Nohara N, et al. Effects of acetate-free citrate dialysate on glycoxidation and lipid peroxidation products in hemodialysis patients. Nephron Extra. 2012 Jan;2(1):256-68.

40. Gabutti L, Lucchini B, Marone C, Alberio L, Burnier M. Citrate- vs. acetate-based dialysate in bicarbonate haemodialysis: consequences on haemodynamics, coagulation, acid-base status, and electrolytes. BMC Nephrol. 2009 Mar 5;10:7.

41. Grundström G, Christensson A, Alquist M, Nilsson L-G, Segelmark M. Replacement of acetate with citrate in dialysis fluid: a randomized clinical trial of short term safety and fluid biocompatibility. BMC Nephrol. 2013 Oct 9;14:216.

42. Schmitz M, Loke O, Fach B, Kalb K, Heering PJ, Meinke D, et al. Effects of citrate dialysate in chronic dialysis: a multicentre randomized crossover study. Nephrol Dial Transplant. 2016;31(8):1327-34.

43. Hyšpler R, Tichá A, Šafránek R, Moučka P, Nývltová Z, Štochlová K, et al. Indoxyl sulfate elimination in renal replacement therapy: influence of citrate- versus acetate-buffering component during bicarbonate dialysis. Dis Markers. 2018;2018:3985861.

44. Jung SW, Kim DR, Cho KS, Seo J-W, Moon H, Park EJ, et al. Effects of dialysate acidification with citrate versus acetate on cell damage, uremic toxin levels, and inflammation in 
patients receiving maintenance hemodialysis. Am J Kidney Dis. 2018; doi:

10.1053/j.ajkd.2018.09.010.

45. Jhawar S, Singh P, Torres D, Ramirez-Valle F, Kassem H, Banerjee T, et al. Functional genomic analysis identifies indoxyl sulfate as a major, poorly dialyzable uremic toxin in end-stage renal disease. PloS One. 2015;10(3):e0118703.

46. Lohr JW, Schwab SJ. Minimizing hemorrhagic complications in dialysis patients. J Am Soc Nephrol. 1991 Nov;2(5):961-75.

47. Kishimoto TK, Viswanathan K, Ganguly T, Elankumaran S, Smith S, Pelzer K, et al. Contaminated heparin associated with adverse clinical events and activation of the contact system. $\mathrm{N}$ Engl J Med. 2008 Jun 5;358(23):2457-67.

48. Sands JJ, Kotanko P, Segal JH, Ho C-H, Usvat L, Young A, et al. Effects of citrate acid concentrate (citrasate ${ }^{\circledR}$ ) on heparin $\mathrm{N}$ requirements and hemodialysis adequacy: a multicenter, prospective noninferiority trial. Blood Purif. 2012;33(1-3):199-204.

49. Rocha AD, Padua VC, Oliveira E, Guimaraes MM, Lugon JR, Strogoff de Matos JP. Effects of citrate-enriched bicarbonate based dialysate on anticoagulation and dialyzer reuse in maintenance hemodialysis patients. Hemodial Int. 2014 Apr;18(2):467-72.

50. Leung KCW, Tai DJ, Ravani P, Quinn RR, Scott-Douglas N, MacRae JM. Citrate vs. acetate dialysate on intradialytic heparin dose: A double blind randomized crossover study. Hemodial Int. 2016;20(4):537-47.

51. Šafránek R, Moučka P, Vávrová J, Palička V, Pavlíková L, Sulková SD. Changes of serum calcium, magnesium and parathyroid hormone induced by hemodialysis with citrate-enriched dialysis solution. Kidney Blood Press Res. 2015;40(1):13-21.

52. Daimon S, Dan K, Kawano M. Comparison of acetate-free citrate hemodialysis and bicarbonate hemodialysis regarding the effect of intra-dialysis hypotension and post-dialysis malaise. Ther Apher Dial. 2011 Oct;15(5):460-5.

53. Durand P-Y, Menoyo-Calonge V, Muresan C. FP500 Citrate-based dialysate and muscle cramps: a significant proportion of dialysis patients could have a reduced citrate metabolizing capacity [résumé] Nephrol Dial Transplant. 2015 May 1;30(suppl_3):iii238-9.

54. Durand P-Y, Nicco C, Serteyn D, Attaf D, Edeas M. Microbiota quality and mitochondrial activity link with occurrence of muscle cramps in hemodialysis patients using citrate dialysate: a pilot study. Blood Purif. 2018;46(4):301-8. 
55. Basile C, Rossi L, Lomonte C. The choice of dialysate bicarbonate: do different concentrations make a difference? Kidney Int. 2016 May;89(5):1008-15.

56. Abramowitz MK. Bicarbonate balance and prescription in ESRD. J Am Soc Nephrol. 2017 Mar;28(3):726-34.

57. Kuragano T, Kida A, Furuta M, Yahiro M, Kitamura R, Otaki Y, et al. Effects of acetate-free citrate-containing dialysate on metabolic acidosis, anemia, and malnutrition in hemodialysis patients. Artif Organs. 2012 Mar;36(3):282-90.

58. Pizzarelli F, Cerrai T, Dattolo P, Ferro G. On-line haemodiafiltration with and without acetate. Nephrol Dial Transplant. 2006 Jun;21(6):1648-51.

59. Aniort J, Petitclerc T, Créput C. Safe use of citric acid-based dialysate and heparin removal in postdilution online hemodiafiltration. Blood Purif. 2012;34(3-4):336-43.

60. Molina Nuñez M, de Alarcón R, Roca S, Álvarez G, Ros MS, Jimeno C, et al. Citrate versus acetate-based dialysate in on-line haemodiafiltration. A prospective cross-over study. Blood Purif. 2015;39(1-3):181-7.

61. Panichi V, Fiaccadori E, Rosati A, Fanelli R, Bernabini G, Scatena A, et al. Post-dilution on line haemodiafiltration with citrate dialysate: first clinical experience in chronic dialysis patients. Sci World J 2013;2013:703612.

62. Richtrova P, Mares J, Kielberger L, Trefil L, Eiselt J, Reischig T. Citrate-buffered dialysis solution (citrasate) allows avoidance of anticoagulation during intermittent hemodiafiltration-at the cost of decreased performance and systemic biocompatibility. Artif Organs. 2017 Aug;41(8):759-66. 


\section{Légende de la figure}

Figure 1. Métabolisme de l'acétate. Lorsque que les apports en acétate dépassent le taux de conversion de l'acétate en bicarbonate, une partie de l'acétate en excès se conjugue au coenzyme A, formant l'acétyl CoA impliqué dans de multiples voies métaboliques. Cette conjugaison nécessite l'hydrolyse d'ATP en AMP cyclique (AMPc) par l'adénylate cyclase. L'AMPc active la NO synthase qui catalyse la transformation de L-arginine en L-citrulline + NO. Le NO active des guanylyl-cyclases qui augmentent le taux de GMP cyclique (GMPc) dans les cellules réceptrices. L'augmentation de GMPc active des protéines kinases GMPc-dépendantes (GMPc), responsables du relâchement des cellules musculaires lisses, d'une dépression myocardique et d'une hypotension artérielle. L'acétate stimule également la sécrétion d'interleukine 1 (IL-1), impliquée dans l'inflammation, l'activation des cellules lymphocytaires et la stimulation de la NO synthase inductible. 
Multiples voies métaboliques :

-Cycle de Krebs

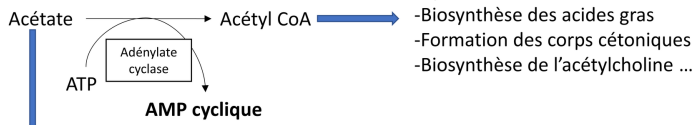

Inflammation

Activation

Iymphocytaire

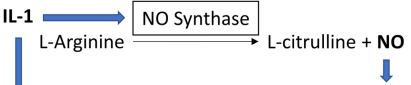

Activation des guanylyl-cyclases

GMP cycliques

Activation PKG :

-Relâchement des cellules musculaires lisses

-Dépression myocardique

-Hypotension artérielle 
Tableau 1 - Principales études comparant les dialysats au bicarbonate acidifiés à l'acide citrique et les dialysats au bicarbonate acidifiés à l'acide acétique en hémodialyse.

\begin{tabular}{|c|c|c|c|c|}
\hline $\begin{array}{l}\text { Référ } \\
\text { ence }\end{array}$ & Description de l'étude & $n$ & Résultats cliniques & Résultats biologiques \\
\hline [38] & $\begin{array}{l}\text { - Prospective, randomisée, } \\
\text { en cross-over } \\
\text { - } 1 \text { séance de dialyse avec } \\
\text { AcDi contre } 1 \text { séance de } \\
\text { dialyse avec CiDi }\end{array}$ & 74 & $\mathrm{~N} / \mathrm{A}$ & $\begin{array}{l}\text { Fin de séance : } \\
\text { - calcémie totale, } \mathrm{Ca}^{2+} \text { ionisée et } \\
\text { magnésémie plus basses avec } \\
\text { citrate qu'avec acétate }(p<0.01 \text { ) } \\
\text { - Citratémie plus élevée avec CiDi } \\
\text { ( } p=0.01) \\
\text { - Acidose, } \triangle \text { urée : NS }\end{array}$ \\
\hline [38] & $\begin{array}{l}\text { - "Exclusive-use study»: } \\
12 \text { semaines } \\
\text { d'hémodialyse avec CiDi } \\
\text { - Paramètres biologiques au } \\
\text { début et à la fin des } 12 \\
\text { semaines. }\end{array}$ & 25 & $N / A$ & $\begin{array}{l}\text { Entre début et fin de l'étude : } \\
\text { - } \nearrow \mathrm{Kt} / \mathrm{V}(\mathrm{p}=0,01) \text { et taux de } \\
\text { réduction de l'urée }(p<0,03) \\
\text { - début de séance : } \searrow \\
\text { magnésémie }(p<0.05) ; \nearrow \text { réserve } \\
\text { alcaline }(p<0.05) ; \text { calcémie } \\
\text { totale, } \mathrm{Ca}^{2+} \text { ionisée, } \mathrm{pH}: \mathrm{NS}\end{array}$ \\
\hline [40] & $\begin{array}{l}\text { Prospective, randomisée, } \\
\text { contrôlée, en cross-over } \\
\text { CiDi et AcDi en alternance } \\
\text { (4 semaines en tout) puis } \\
\text { CiDi enrichi avec Ca 0,25 } \\
\text { mmol/L pendant } 1 \\
\text { semaine }\end{array}$ & 25 & $\begin{array}{l}\searrow \text { PA systolique, } P A \\
\text { diastolique }(p<0,01) \text { et } \\
\text { résistances } \\
\text { périphériques }(p<0,001 \\
\text { ) avec CiDi } \\
\text { Enrichissement en Ca: } \\
\text { corrige } \searrow \text { PA mais pas }\end{array}$ & $\begin{array}{l}\text { - Kt/ }, \text {, coagulation : } \mathrm{NS} \\
\text { - début de séance : } \mathrm{Ca}^{2+} \mathrm{NS}, \nearrow \\
\text { réserve alcaline avec } \mathrm{CiDi} \\
(\mathrm{p}<0,01) \\
\text { - Fin de séance }: \searrow \mathrm{Ca}^{2+} \text { et } \searrow \mathrm{pH} \\
\text { avec } \mathrm{CiDi}\end{array}$ \\
\hline
\end{tabular}




\begin{tabular}{|c|c|c|c|c|}
\hline & $\begin{array}{l}\text { - Objectif primaire : } \\
\text { tolérance } \\
\text { - Objectif secondaire : } \\
\text { efficacité }\end{array}$ & & $\begin{array}{l}\searrow \text { résistances } \\
\text { périphériques }\end{array}$ & \\
\hline $\begin{array}{l}\text { [37] } \\
\end{array}$ & 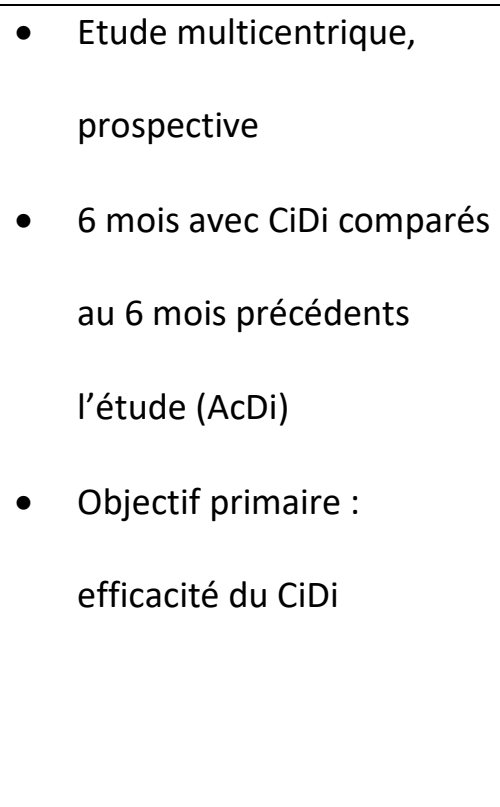 & & & $\begin{array}{l}-\lambda \mathrm{Kt} / \mathrm{V}(\mathrm{p}<0.0001) \text { avec CiDi } \\
-\searrow \beta 2 \text { microglobulinémie } \\
(p<0,0001) \text { avec CiDi } \\
\text { Début de séance: } \\
-\searrow \text { réserve alcaline avec CiDi } \\
(\text { CiDi : } 22,9 \pm 2,9 \text { contre AcDi : } \\
24,6 \pm 3,4, p<0,0001) \\
-\searrow \text { aluminium sérique avec CiDi } \\
(p<0,001)\end{array}$ \\
\hline [48] & $\begin{array}{l}\text { Etude multicentrique, } \\
\text { prospective } \\
4 \text { périodes d'étude de } 2 \\
\text { semaines chacune : } \\
\text { (B) = AcDi + } 100 \% \text { héparine } \\
\text { (P1) = CiDi + } 100 \% \text { héparine } \\
\text { (P2) = CiDi + } 80 \% \text { héparine } \\
\text { (P3) = CiDi + } 66.7 \% \text { héparine } \\
\text { Objectif primaire : non- } \\
\text { infériorité (P2) par rapport } \\
\text { à (B) quant à l'efficacité de } \\
\text { la dialyse. }\end{array}$ & 277 & $\begin{array}{l}\text { - Plus de crampes avec } \\
\text { CiDi. Par rapport à (B) : } \\
(\mathrm{P} 1)+2,4 \%,(\mathrm{P} 2)+0,9 \% \text {, } \\
(\mathrm{P} 3)+0,5 \% \\
\text { - Pas plus d'événements } \\
\text { thrombotiques après } \\
\text { réduction des doses } \\
\text { d'héparine avec CiDi }\end{array}$ & $\begin{array}{l}\text { - Objectif primaire atteint } \\
\text { - Fin de séance }: \searrow \text { calcémie } \\
\text { totale et } \mathrm{Ca}^{2+} \text { ionisée avec CiDi }\end{array}$ \\
\hline
\end{tabular}




\begin{tabular}{|c|c|c|c|c|}
\hline [41] & 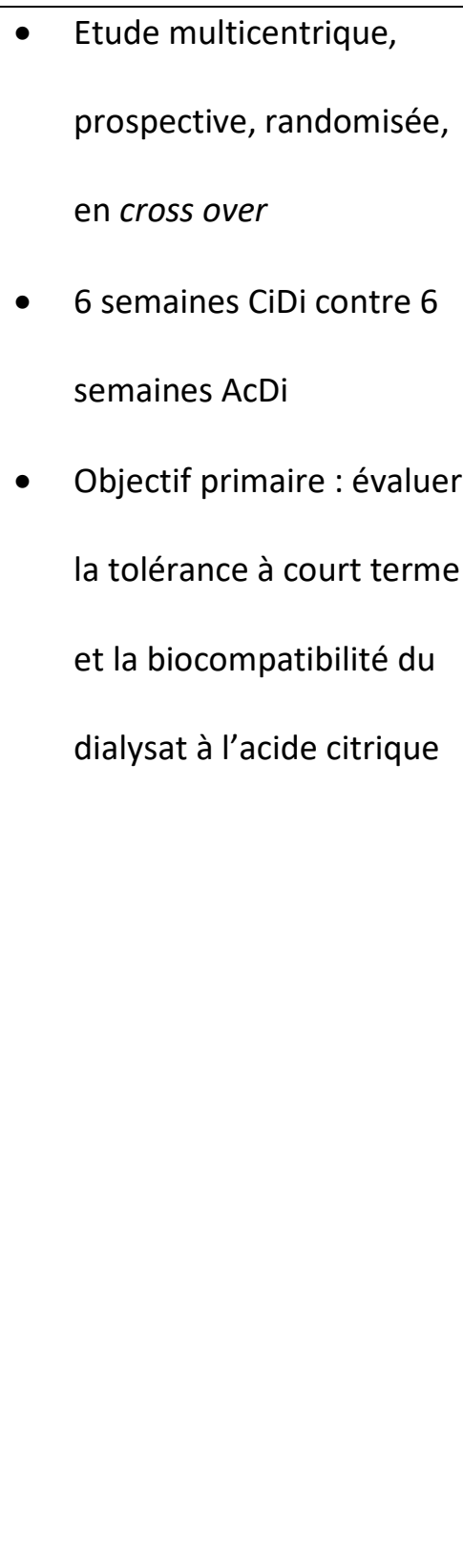 & 20 & & $\begin{array}{l}\text { - Kt/V : NS } \\
-\checkmark \text { TA à H1 et H3 avec CiDi } \\
\text { (p<0,05) } \\
\text { - } \nearrow \text { citratémie pendant la séance } \\
\text { (p<0,0001) } \\
\text { - Ca2+ ionisée : } \nearrow \text { pendant la } \\
\text { dialyse avec AcDi (de } 1,14 \pm 0,02 \\
\text { à 1,27 } \pm 0,01, p<0,0001 \text { ), } \searrow \\
\text { pendant la dialyse avec CiDi (de } \\
1,14 \pm 0,02 \text { à } 1,10 \pm 0,01, \\
p<0,0001 \text { ) } \\
\text { - [PTH] : stable pendant la dialyse } \\
\text { avec CiDi, } \searrow \text { pendant la dialyse } \\
\text { avec AcDi ( } p<0,001 \text { ) } \\
\text { - PTX3 : augmente moins pendant } \\
\text { la dialyse avec CiDi ; autres } \\
\text { du stress oxydatif : NS }\end{array}$ \\
\hline [42] & $\begin{array}{l}\text { - Etude multicentrique, } \\
\text { prospective, randomisée, } \\
\text { en cross over } \\
\text { - } 4 \text { semaines CiDi contre } 4 \\
\text { semaines AcDi } \\
\text { Objectif primaire (safety } \\
\text { assessement) : évaluer le }\end{array}$ & 48 & $\begin{array}{l}7 \text { significative fatigue, } \\
\text { crampes, douleurs } \\
\text { musculaires avec CiDi } \\
\text { (surtout les } 2 \text { premières } \\
\text { semaines) }\end{array}$ & $\begin{array}{l}\text { - Kt/ } \mathrm{V} \text {, taux de réduction de } \\
\text { I'urée, taux de réduction de la } \\
\text { créatinine, de la } \beta 2- \\
\text { microglobuline : NS } \\
\text { - Fin de séance: Ca }{ }^{2+} \text { ionisée, } \\
\text { réserve alcaline et magnésémie } \\
\text { plus basses avec CiDi qu'avec } \\
\text { AcDi ( } p<0.05),[P T H] \text { plus élevée }\end{array}$ \\
\hline
\end{tabular}




\begin{tabular}{|l|l|l|l|}
\hline $\begin{array}{l}\text { déséquilibre de la balance } \\
\text { calcique et du statut } \\
\text { acidobasique }\end{array}$ & & & avec CiDi qu'avec AcDi (p<0.001). \\
$\qquad \begin{array}{l}\text { Objectif secondaire : } \\
\text { efficacité }\end{array}$ & & & \\
\hline
\end{tabular}

AcDi : dialysat au bicarbonate acidifié à l'acide acétique (4 à $8 \mathrm{mmol} / \mathrm{L}$ ) ; CiDi : dialysat au bicarbonate acidifié à l'acide citrique $(0,8$ à $2,4 \mathrm{mEq} / \mathrm{L}$ avec ou sans acide acétique $0,3 \mathrm{mmol} / \mathrm{L}) ; \mathrm{N} / \mathrm{A}$ : non rapportés ; NS : pas de différence significative ; PA = pression artérielle ; PTH : parathormone ; CRP : protéine $\mathrm{C}$ réactive. 